\title{
Berkeley Center for Synthetic Biology
}

The world's first synthetic biology department at the Lawrence Berkeley National Laboratory is using a bottom-up approach to form a foundation of design rules and models to understand cellular function.

Bringing together the fields of engineering, the physical sciences and the biological sciences, synthetic biology lends credence to Carlos Bustamante's idea that "we are becoming scientists-not engineers, physicists, chemists and biologists." Bustamante is in the Department of Biochemistry, Molecular Biology and Physics at the University of California, Berkeley and is department head in the Physical Biosciences Division (PBD) at Lawrence Berkeley National Laboratory (LBNL). According to PBD faculty scientist and Department of Bioengineering Associate Professor Adam Arkin, "Cell and molecular biology has been moving up toward engineering, and engineering as an organismal science is moving down to cells, and synthetic biology is where they're meeting."

Graham Fleming, deputy laboratory director of the PBD and UC Berkeley professor of chemistry, had a vision of bringing together researchers who were very physically and quantitatively minded, and who could start to dissect cellular networks and individual molecular machines up to the whole cell. As the PBD faculty were learning how to reverse engineer systems for new behaviors, it became clear that a focused effort under the auspices of a National Laboratory would prove useful in taking the new knowledge "beyond the episodic discovery of a principle and really turn it into an engineering discipline," according to Arkin. Taking advantage of their extensive collaboration with and physical proximity to UC Berkeley, the PBD established the world's first synthetic biology department under the direction of Jay Keasling, a professor of chemical engineering and bioengineering at UC Berkeley and also the division director of the PBD. It has started as a somewhat self-assembled group of Berkeley and LBNL faculty but will more than double to upwards of ten faculty scientists in the next few years.

Having been recently awarded a $\$ 500$ million grant from British Petroleum, one of the world's biggest energy companies, LBNL has secured its reputation as a pioneer of large-scale efforts. LBNL has already seen success with Keasling's artemisinin project, which used synthetic biology to generate microbial 'factories' to produce artemisinic acid, a precursor to the antimalarial drug artemisinin, which is currently unattainable to the world's poorest children because of the laborious and expensive procedures required to extract it from its natural sources. Having taken 100 person years to complete, the artemisinin project serves as a reminder of how difficult synthetic biology initiatives can be without the proper tools. Indeed, Arkin noted that "it was the later stages of the artemisinin project where there was a great deal of sophistication in the use of modular design and in reusable 'parts' that have been a hallmark of the emerging synthetic biology field."

Creating modular cellular 'parts' to expand on the collection available via the Massachusetts Institute of Technology's Registry of Standard Biological Parts (http://parts.mit.edu/registry/index.php/Main_Page) is a major focus of the new department. The so-called parts fab, or parts fabrication approach, borrows from a concept that exists in microelectronics that Keasling describes as "black boxes." These boxes contain individual well-characterized 'parts' and can be modularly combined without concern for what is contained within them. Synthetic biology black boxes are devices (such as a repressor) that contain individual proteins, such as a DNA binding protein. To get to a point where they can generate a "stripped-down chassis that anybody can use to produce things," Keasling says that they are "thinking carefully up front about which parts are going to be most useful." Arkin notes that the proposed bioenergy project, in which they hope to create a renewable biofuel, will drive which of the infinite number of 'parts' will be developed first.

According to Arkin, "Synthetic biology is nothing more than quantitative biology and genetic engineering except that it has a central goal to make things cheaper, predictable, reliable and scalable up to large systems." Indeed, from its origins in biomedical engineering, bioengineering and biological engineering, Fletcher hopes that "synthetic biology will become a relatively broad term to reflect various people's efforts to engineer biological systems to have a behavior," distinguishing synthetic biology from basic biology in the sense that the former will not be as oriented towards understanding a system, but instead will focus on using biological components to creatively solve problems. However, understanding the biological

"Synthetic biology will
become a relatively
broad term to reflect
various people's efforts
to engineer biological
systems to have a
behavior."

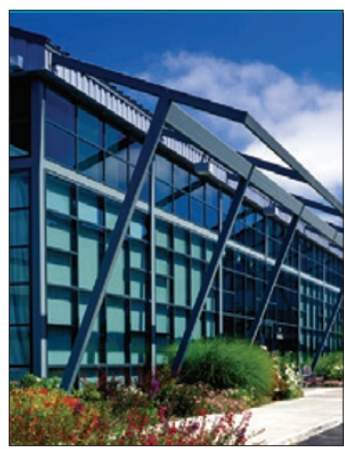

system will be an important synthetic biology side effect: Fletcher notes that "if we try to design a system and we fail, it's probably because we didn't understand it."

Coming from an engineering and applied physics background, Fletcher says that he is inclined to "do things because they are useful" - though he appreciates the satisfaction of understanding a system. His lab is working on various aspects of how cells generate forces and respond to forces by reconstituting actin networks de novo. He believes that generating complex behavior by putting various simple biological systems together is the characteristic that distinguishes synthetic biology from engineering and is a huge benefit to the bottom-up approach. Though building a new biological machine (such as a new cell from its component parts) may raise numerous ethical and security questions, Bustamante suggests that "by generating a minimal organism, we are essentially asking the question of what the rules really are that underlie the living state: what makes living systems living?" His lab would like to build an artificial, minimal cell starting from the rudimentary mitochondrion and adding back components that will make it first transcriptionally independent and then replicationally independent. He notes that "it leads to this idea that you can use it as a chassis to build a complex cell, which is one of the theories we are trying to demonstrate."

Keasling notes the difficulty that some biologists have in embracing this idea of the black box: "This is a different frame of mind." According to Bustamante, some of this may sound like science fiction at this point, "but we need to start somewhere. The ideas are crazy, and some of these ideas will go down the drain and others will survive. But, hopefully other people will get inspired to play these types of games. That is what science is all about."

Mirella Bucci, Berkeley, California 